\title{
A Rare Case of Euphoria Caused by Lidocaine After an Erector Spinae Plane Block: A Case Report
}

This article was published in the following Dove Press journal: Journal of Pain Research

\section{Joo Yong Lee (D) \\ Hyeon Tae Kim 10 \\ Jeong Moon Won (D) \\ Young Duck Shin (D)}

Department of Anesthesiology and Pain Medicine, Chungbuk National University Hospital, College of Medicine, Chungbuk National University, Cheongju, Korea
Correspondence: Young Duck Shin; Hyeon Tae Kim

Department of Anesthesiology and Pain Medicine, Chungbuk National University Hospital, 776, I Sunhwan-Ro, Seowon-

$\mathrm{Gu}$, Cheongju, Chungbuk 28644, Korea

Tel +82-43-269-6234; +82-43-269-6988

Fax +82-43-272-0264

Email yydshin@naver.com;

gskht@naver.com

\begin{abstract}
Lidocaine is a cost-effective drug that is widely used for local and regional anesthesia. However, central nervous system (CNS) toxicity can occur when lidocaine is administered above the maximum recommended dose (approximately $4.5 \mathrm{mg} / \mathrm{kg}$ ) or if lidocaine is injected intravascularly rather than administered locally. Systemic toxicity by lidocaine has been reported in several studies. However, psychotic reactions due to lidocaine have been rarely reported; furthermore, reports of lidocaine-related euphoria are very rare. We report a very rare case of euphoria caused by CNS toxicity that occurred during the local administration of lidocaine at the therapeutic dose. Therefore, anesthesiologists should be aware of the severe side effects of local anesthetics despite administering the appropriate dosage at the appropriate location. Future studies should investigate pharmacokinetics to determine the safety profile of local anesthetics.
\end{abstract}

Keywords: euphoria, lidocaine, adverse effects, nerve block

\section{Introduction}

Local anesthetics account for 5-10\% of all side effects reported for anesthetic drugs. ${ }^{1}$ Side effects include cardiovascular and central nervous system (CNS) toxicity due to blockage of cell membrane ion channels, peripheral nerve complications due to other effects of the drug or the vehicle, allergic reactions, and needle trauma or infection due to mechanical or other technical problems. ${ }^{1}$ The incidence of toxicity caused by local anesthetics occurs more often by accidental intravascular injection than by overdosage; nevertheless, the arterial blood concentration of the local anesthetic is an important factor in both cases. ${ }^{2}$

A higher dosage of lidocaine in the stellate ganglion block and epidural block results in a higher blood concentration. ${ }^{3,4}$ Therefore, if local anesthetic-induced systemic toxicity occurs, overdosage should be considered during both intravascular administration and local administration. However, due to the lack of quality data, specific recommendations for the general maximum dose of lidocaine cannot be determined, and the manufacturer's recommended dose varies from country to country. ${ }^{5}$ According to most manufacturer recommendations, the maximum lidocaine dose for local anesthesia and local nerve block is $300 \mathrm{mg}$ for a patient weighing $70 \mathrm{~kg}$ (approximately $4.5 \mathrm{mg} / \mathrm{kg}$ ). ${ }^{5}$

First described by Forero et $\mathrm{al}^{6}$ in 2016 for thoracic neuropathic pain, the erector spinae plane block (ESPB) is a novel technique of interfascial plane block between 
the transverse process and the erector spinae muscle. The erector spinae muscle is highly vascularized, ${ }^{7}$ and during the ESPB procedure, a large surface area of the muscle comes into contact with local anesthetics. ${ }^{8}$ However, it has been reported that even with a dose of $0-3.72 \mathrm{mg} / \mathrm{kg}$ lidocaine, serum lidocaine concentrations did not reach the systemic toxicity range, ${ }^{9}$ and there are few reports of systemic toxicity symptoms due to local anesthetics used for ESPB.

Side effects of lidocaine have been reported in several studies ${ }^{10-12}$ and have been known to occur during intravascular injection or overdosage.$^{13}$ However, side effects, such as psychotic reactions, particularly euphoria, have rarely been reported. We report a rare case of euphoria caused by lidocaine after an ESPB.

\section{Case Report}

This case was approved by the Chungbuk National University Hospital Clinical Research Review Committee (approval number 2020-04-010-001). The patient provided informed consent for the publication of this case report.

A 48-year-old man $(164 \mathrm{~cm}, 79 \mathrm{~kg})$ visited the pain clinic for herpes zoster pain. The patient had blisters on the left flank area (left T6 area) that developed three days ago. The patient reported pain that was localized to his left flank (rated 5-7/10 on a numeric rating scale) accompanied by a burning and tingling sensation. The patient previously received a caudal block to treat back pain due to spondylolytic spondylolisthesis at L5/S1 and an adductor canal block to treat left knee pain with lidocaine; no side effects were observed during these procedures.

An ESPB was scheduled to treat the patient's pain as the first treatment. The patient did not take any medication or addictive drug (such as cocaine) that may interact and potentially increase serum levels and potential for toxicity. The patient had no psychosis or other psychological disorders.

After disinfection, the patient was placed in the prone position, and a 22-gauge quince needle was inserted under ultrasound guidance in plane towards the transverse process T6 level. We confirmed the needle location in the fascial plane between the transverse process and erector spinae muscle under ultrasound guidance. After confirming that blood was not aspirated, $10 \mathrm{~mL}$ of $1 \%$ lidocaine and $10 \mathrm{~mL}$ of normal saline was injected. Lidocaine was slowly injected in $5 \mathrm{~mL}$ increments at a rate of $1 \mathrm{~mL}$ every 3 seconds with a careful repetitive aspiration to avoid intravascular injection. The procedure was completed after confirming separation of the erector spinae muscles from the transverse process with a good caudal and cephalic spread.

Two minutes after the procedure, the patient began to laugh, saying inaccurately that he was feeling very good. We could not identify the cause of the patient's symptoms except for the lidocaine injected into the erector spinae plane.

The patient's vital signs (electrocardiogram, oxygen saturation, tidal $\mathrm{CO}_{2}$, etc) remained within the normal limit, and intubation and emergency kits were prepared. Except for slurred speech and euphoria, the patient had no neurological symptoms, such as numbness of the tongue or lip, lightheadedness, tinnitus, or visual disturbance. After 35 minutes, the patient's neurological symptoms improved completely, and accurate communication was possible. Dermatomal coverage after the procedure with pinprick and/or cold test was not performed. The patient's left flank pain decreased to a numerical rating scale score of 1-2/10 after ESPB and remained so for over 2 hours until discharge. He remembered exactly what was going on during and after the procedure. He said, "I seemed to be drunk. It was the most pleasant and happy feeling I have ever had." The patient was observed for 2 hours, and after confirming that there were no neurological symptoms, he was discharged.

\section{Discussion}

Among the adverse events caused by CNS toxicity due to lidocaine, psychotic reactions have been previously reported. ${ }^{10-12}$ Symptoms of psychotic reactions were mainly mood changes, doom anxiety, hallucinations, and delusions, ${ }^{11}$ but euphoria has been rarely reported. Shortlasting euphoria after intravenous lidocaine administration was reported in a 42-year-old patient who was addicted to cocaine. ${ }^{12}$ Euphoria was also reported after administration of $35 \mathrm{~mL}$ of $2 \%$ lidocaine for axillary block and $50 \mathrm{~mL}$ of $1 \%$ lidocaine for local anesthesia. ${ }^{10}$ In both cases, euphoria occurred after intravenous administration with a high possibility of CNS toxicity or after administration of a dose higher than $300 \mathrm{mg}$ of lidocaine. ${ }^{10,12}$ However, our case differs from these cases since euphoria occurred during the local, not intravascular administration of lidocaine and at a dose lower ( $10 \mathrm{~mL}$ of $1 \%$ lidocaine) than the maximum recommended dose. This suggests that CNS toxicity of local anesthetics may occur during local administration, even at doses lower than the known maximum 
dose. The characteristics of ESPB and the patient's position can be considered as the reasons these side effects occurred with ESPB.

Although randomized, controlled studies have not been performed yet, the efficacy of ESPB for acute herpes pain has been demonstrated in several studies. ${ }^{14,15}$ The mechanism of action is unclear, but it has been reported that the injected local anesthetic spreads through the costotransverse foramen to the external intercostal muscle and internal intercostal membrane, ${ }^{6}$ and interfacial injected local anesthetics spread through the costotransverse foramen and peripheral porous tissues to the intercostal and thoracic paravertebral spaces. ${ }^{16}$ Additionally, sensitivity depends on the integrity of A-gamma fibers (light touch), A-delta fibers (cold and pinprick), and $\mathrm{C}$ fibers (warmth and dull pain). ${ }^{17}$ Several studies reported that the analgesic effect of ESPB is due to a differential block mediated by non-myelinated $\mathrm{C}$ fibers rather than larger A-delta and A-gamma fibers. ${ }^{18,19}$

Elkoundi et al reported that plasma local anesthetic levels are expected to increase significantly when large amounts of local anesthetics are administered with ESP blockade. $^{20}$ The peak plasma concentration of a local anesthetic is determined by the vascular supply-dependent rate of systemic absorption at the injection site and surface area contact with the local anesthetic injectate. ${ }^{21}$ When ESPB is performed, a large surface area comes into contact with local anesthetics. ${ }^{8}$ Furthermore, the erector spinae muscle is highly vascularized. ${ }^{7}$ Considering these mechanisms of ESPB and the blood supply of erector spinae musculature, lidocaine was likely rapidly absorbed over a large area in a short time, and the intramuscular or interfacial spread of the lidocaine may have caused a rapid increase in the level of lidocaine in the blood. Therefore, interfacial administration of local anesthetics could cause CNS toxicity to a greater degree than typical local administration. However, Caruso et al reported that serum lidocaine concentrations were below the systemic toxicity range, even with an injection of $0-3.72 \mathrm{mg} / \mathrm{kg}$ of lidocaine for ESPB in a study of 27 patients. ${ }^{9}$ In this regard, future pharmacokinetic studies of local anesthetics in ESPB are needed to determine their safety profile.

Another possible cause of euphoria was the patient's position during the procedure. The concentration of local anesthetics reaching the brain depends on the ratio of cardiac output to the brain. ${ }^{2}$ The patient's prone position during the procedure may have led to euphoria due to the increased cardiac output to the brain. Therefore, the patient's prone position during the procedure could also be a factor that could affect CNS toxicity by local anesthetics.

The reported sequence of CNS toxicity symptoms after local anesthetic injection is numbness of the tongue, lightheadedness, tinnitus, visual disturbance, slurring of speech, muscular twitching, irrational conversation, unconsciousness, convulsion, coma, and apnea. ${ }^{2}$ Furthermore, medical professionals usually assess numbness of the tongue, tinnitus, and visual disturbance to evaluate CNS toxicity caused by local anesthetic. However, in our case, the first symptom was euphoria and slurring of speech. This indicates that symptoms of CNS toxicity may occur in a different order than that previously reported or even skip steps in this sequence. Thus, we speculate that any sequence, including the initial sequence of CNS toxicity symptoms, can be a predicting factor for a life-threatening situation such as seizure, coma, and respiratory arrest.

The limitation of our case report is that laboratory tests were not performed since the patient's symptoms completely disappeared within 35 minutes after the procedure. Performing laboratory tests on patients before and after the procedure could help identify other factors that cause lidocaine-induced systemic toxicity. In this case, adrenaline was not added to lidocaine, but it is worth considering adding adrenaline to local anesthetics to avoid the side effects of local anesthetics. The addition of a small amount of adrenaline to a local anesthetic acts as a marker of intravascular absorption and significantly reduces the peak plasma concentration of the local anesthetic, reducing systemic toxicity. $^{22}$

\section{Conclusion}

In summary, euphoria is a very rare side effect of systemic toxicity that can occur after lidocaine injection. The occurrence of this side effect with ESPB suggests that future pharmacokinetic studies are needed to determine the safety profile. Furthermore, the severe side effects of local anesthetics should be considered despite administering the appropriate dose at the appropriate location while avoiding intravascular administration. In addition, the patient's position during the procedure may also cause unexpected side effects.

\section{Acknowledgments}

We would like to thank Editage (www.editage.com) for English language editing. 


\section{Author Contributions}

All authors made a significant contribution to the work reported, whether that is in the conception, study design, execution, acquisition of data, analysis and interpretation, or in all these areas; took part in drafting, revising or critically reviewing the article; gave final approval of the version to be published; have agreed on the journal to which the article has been submitted; and agree to be accountable for all aspects of the work.

\section{Funding}

No funding was received for this report.

\section{Disclosure}

The authors declare that they have no competing interests for this work.

\section{References}

1. McCaughey W. Adverse effects of local anaesthetics. Drug Saf. 1992;7 (3):178-189. doi:10.2165/00002018-199207030-00003

2. Scott DB. Toxic effects of local anaesthetic agents on the central nervous system. Br J Anaesth. 1986;58(7):732-735. doi:10.1093/bja/ 58.7.732

3. Park HS, Chung CJ, Chin YJ. The plasma concentrations and systemic toxicity of lidocaine after maximal or supramaximal recommended doses of epidural administration. Korean J Pain. 1999;12:36-42.

4. Song SO, Suh YH. Changes of plasma lidocaine concentrations after Stellate Ganglion Block according to volume-changes of $1 \%$ lidocaine. Korean J Pain. 2001;14:26-31.

5. Weinberg L, Peake B, Tan C, Nikfarjam M. Pharmacokinetics and pharmacodynamics of lignocaine: a review. World $J$ Anesthesiol. 2015;4(2):17-29. doi:10.5313/wja.v4.i2.17

6. Forero M, Adhikary SD, Lopez H, Tsui C, Chin KJ. The erector spinae plane block: a novel analgesic technique in thoracic neuropathic pain. Reg Anesth Pain Med. 2016;41:621-627. doi:10.1097/AAP.00000 00000000451

7. Yue BY, le Roux CM, Corlett R, De La Harpe D, Richardson M, Ashton M. The arterial supply of the cervical and thoracic spinal muscles and overlying skin: anatomical study with implications for surgical wound complications. Clin Anat. 2013;26(5):584-591. doi:10.1002/ca.22139
8. Yang HM, Choi YJ, Kwon HJ, O J, Cho TH, Kim SH. Comparison of injectate spread and nerve involvement between retrolaminar and erector spinae plane blocks in the thoracic region: a cadaveric study. Anaesthesia. 2018;73(10):1244-1250. doi:10.1111/anae.14408

9. Caruso TJ, Lin C, O'Connell C, et al. Systemic absorption of lidocaine from continuous erector spinae plane catheters after congenital cardiac surgery: a retrospective study. $J$ Cardiothorac Vasc Anesth. 2020;S1053-0770(20)30502-4. doi:10.1053/j.jvca.2020.05.040.

10. Zeidan A, Baraka A. Is euphoria a side-effect of lidocaine? Anaesthesia. $\quad 2004 ; 59(12): 1253-1254$ doi:10.1111/j.13652044.2004.04025.x

11. Saravay SM, Marke J, Steinberg MD, Rabiner CJ. "Doom anxiety" and delirium in lidocaine toxicity. Am J Psychiatry. 1987;144(2):159-163.

12. Blanke J, Wolstein J, Paulus HJ. Euphoric effect of lidocaine. Psychiatr Prax. 1996;23(2):90-91.

13. Becker DE, Reed KL. Local anesthetics: review of pharmacological considerations. Anesth Prog. 2012;59(2):90-102. doi:10.2344/00033006-59.2.90

14. Aydin T, Balaban O, Ahiskalioglu A, Alici HA, Acar A. Ultrasoundguided erector spinae plane block for the management of herpes zoster pain: observational study. Cureus. 2019;11(10):e5891.

15. Tekin E, Ahiskalioglu A, Aydin ME, Sengun E, Bayramoglu A, Alici HA. High-thoracic ultrasound-guided erector spinae plane block for acute herpes zoster pain management in emergency department. Am J Emerg Med. 2019;37(2):375.e1-375.e3. doi:10.1016/j.ajem.2018.10.028

16. Adhikary SD, Bernard S, Lopez H, Chin KJ. Erector spinae plane block versus retrolaminar block: a magnetic resonance imaging and anatomical study. Reg Anesth Pain Med. 2018;43(7):756-762.

17. Mackenzie RA, Burke D, Skuse NF, Lethlean AK. Fibre function and perception during cutaneous nerve block. J Neurol Neurosurg Psychiatry. 1975;38(9):865-873. doi:10.1136/jnnp.38.9.865

18. Chin KJ, Adhikary SD, Forero M. Understanding ESP and fascial plane blocks: a challenge to omniscience. Reg Anesth Pain Med. 2018;43:807-808. doi:10.1097/AAP.0000000000000857

19. Ford DJ, Raj PP, Singh P, Regan KM, Ohlweiler D. Differential peripheral nerve block by local anesthetics in the cat. Anesthesiology. 1984;60:28-33. doi:10.1097/00000542-198401000-00007

20. Elkoundi A, Balkhi H, Bensghir M, Baite A. Levobupivacaine plasma level between erector spinae plane block and thoracic paravertebral block. Reg Anesth Pain Med. 2020;rapm-2020-101406. doi:10.1136/rapm-2020-101406

21. Yasumura R, Kobayashi Y, Ochiai R. A comparison of plasma levobupivacaine concentrations following transversus abdominis plane block and rectus sheath block. Anaesthesia. 2016;71:544-549. doi:10.1111/anae.13414

22. Karmakar MK, Ho AM, Law BK, Wong AS, Shafer SL, Gin T. Arterial and venous pharmacokinetics of ropivacaine with and without epinephrine after thoracic paravertebral block. Anesthesiology. 2005;103(4):704-711. doi:10.1097/00000542-200510000-00008

\section{Publish your work in this journal}

The Journal of Pain Research is an international, peer reviewed, open access, online journal that welcomes laboratory and clinical findings in the fields of pain research and the prevention and management of pain. Original research, reviews, symposium reports, hypothesis formation and commentaries are all considered for publication. The manuscript
Journal of Pain Research

\section{Dovepress}

management system is completely online and includes a very quick and fair peer-review system, which is all easy to use. Visit http:// www.dovepress.com/testimonials.php to read real quotes from published authors. 